\title{
Medical evaluation and management of urolithiasis
}

\author{
Michelle Jo Semins and Brian R. Matlaga
}

\begin{abstract}
Nephrolithiasis is a highly prevalent condition with a high recurrence rate that has a large impact on the quality of life of those affected. It also poses a great financial burden on society. There have been great advancements in the surgical treatment of stone disease over the past several decades. The evolution of surgical technique appears to have overshadowed the importance of prevention of stone disease despite evidence showing medical therapies significantly decreasing stone recurrence rates. Herein we review the metabolic evaluation of stone formers with the use of specific blood and urine tests. We complete our discussion with a review of the medical management of stone formers providing both general recommendations as well as reviewing focused therapies for specific metabolic abnormalities and medical
\end{abstract} conditions.

Keywords: nephrolithiasis, kidney, calculi, prevention, metabolic evaluation

\section{Introduction}

Approximately $10 \%$ of the United States population is afflicted with kidney stone disease, and lifetime male and female prevalence rates of $13 \%$ and $7 \%$, respectively, have been reported [Heilberg and Schor, 2006; Stamatelou et al. 2003; Levy et al. 1995]. These values have recently been noted to be increasing for both genders, although the rate of rise may be greater for females than for males. Importantly, kidney stones are a recurrent disorder, with lifetime recurrence risks reported to be as high as 50\% [Odvina and Pak, 2007; Prezioso et al. 2007].

Recent decades have witnessed great advances in the surgical treatment of kidney stones. Whereas stones were once treated with invasive open surgical techniques, in the present day almost all urinary calculi are treated either in a completely noninvasive fashion with shockwave lithotripsy or in a minimally invasive endoscopic fashion with ureteroscopy or percutaneous nephrolithotomy [Pearle et al. 2005]. While there have been great advancements in the surgical treatment of stoneforming patients, efforts to prevent the formation of kidney stones with medical therapy have not experienced the same rates of advance. Such a failure is disappointing, as medical therapies have been well demonstrated to significantly decrease stone recurrence rates [Lotan et al. 2005].
For these reasons, a proper understanding of the metabolic evaluation and medical management of a stone former is an important component of a complete urologic practice. Such techniques will often identify patient-specific factors that may be modified in order to reduce the risk for recurrent stone formation [Prezioso et al. 2007; Heilberg and Schor, 2006; Begun et al. 1997; Levy et al. 1995; Preminger, 1994; Pak et al. 1980]. Herein, we review the medical evaluation and therapy of stone formers.

\section{Medical evaluation of stone formers}

\section{Blood tests}

Blood screening tests should be a routine component of the diagnostic evaluation for all stone formers (Table 1). Serum electrolyte, calcium, carbon dioxide, and uric acid measurements should all be obtained as well as measurement of serum creatinine to assess renal function. These tests are generally inexpensive, and will effectively screen for metabolic abnormalities that may contribute to recurrent stone formation.

Primary hyperparathyroidism may manifest with hypercalcemia. This disorder can be confirmed by determining the patient's intact serum parathyroid hormone level and serum phosphorous, as an elevated intact parathyroid hormone level
Ther Adv Urol

(2010) 2(1) 3-9

DOI: $10.1177 /$

1756287210369121

(C) The Author(s), 2010. Reprints and permissions:

http://www.sagepub.co.uk/ journalsPermissions.nav
Correspondence to: Brian R. Matlaga, MD, MPH

James Buchanan Brady Urological Institute, The Johns Hopkins University School of Medicine, 600 North Wolfe Street, Baltimore, MD 21287, USA bmatlagåjhmi.edu

Michelle Jo Semins, MD The James Buchanan Brady Urological Institute, The Johns Hopkins University School of Medicine, Baltimore, MD 21287, USA 
Table 1. Blood tests.

All stone formers
Electrolytes, calcium, potassium, phosphorous
Uric acid, $\mathrm{CO}_{2}$
Creatinine
Vitamin D levels
Circumstantial
Hypercalcemia: intact serum parathyroid hormone
level, phosphorous
Abnormal albumin: ionized calcium
Hyperoxaluria suspected: oxalate levels
Sarcoidosis suspected: angiotensin-converting
enzyme (ACE), calcitriol

and depressed phosphorous supports the diagnosis. Repeat calcium measurements are sometimes necessary for the proper diagnosis of this condition, as hypercalcemia may be intermittent. In addition, ionized calcium values may be helpful, particularly if serum albumin levels are abnormal. Alternatively, if the diagnosis is suspected but the calcium level is normal, the administration of a short course of thiazide-type diuretic can 'unmask' occult cases with resultant hypercalcemia [Eisner et al. 2009].

Distal renal tubular acidosis (RTA) may be suspected in the setting of low potassium and carbon dioxide values. This disorder, part of a spectrum of clinical syndromes of metabolic acidosis, results from a defect in renal tubular hydrogen ion secretion, and therefore is associated with an abnormal urinary acidification process. Serum carbon dioxide is reduced, as a consequence of the chronic metabolic acidosis, which also results in decreased tubular potassium reabsorption. Patients with distal RTA generally form calcium phosphate stones [Evan et al. 2007].

Elevated serum uric acid levels may suggest a gouty diathesis. These patients will commonly form stones composed of uric acid, although in rare situations calcium oxalate stone formation may also occur. In some cases of hyperuricemia, patients may manifest symptoms of gouty arthritis, as well as symptoms from their stones.

Less-commonly encountered conditions may require alternative blood tests for diagnosis. Elevated serum oxalate levels and vitamin D levels can diagnose primary hyperoxaluria and hypervitaminosis $\mathrm{D}$, respectively, both conditions that can be associated with urinary calculi. Sarcoidosis can also be associated with
Table 2. Urinary tests.

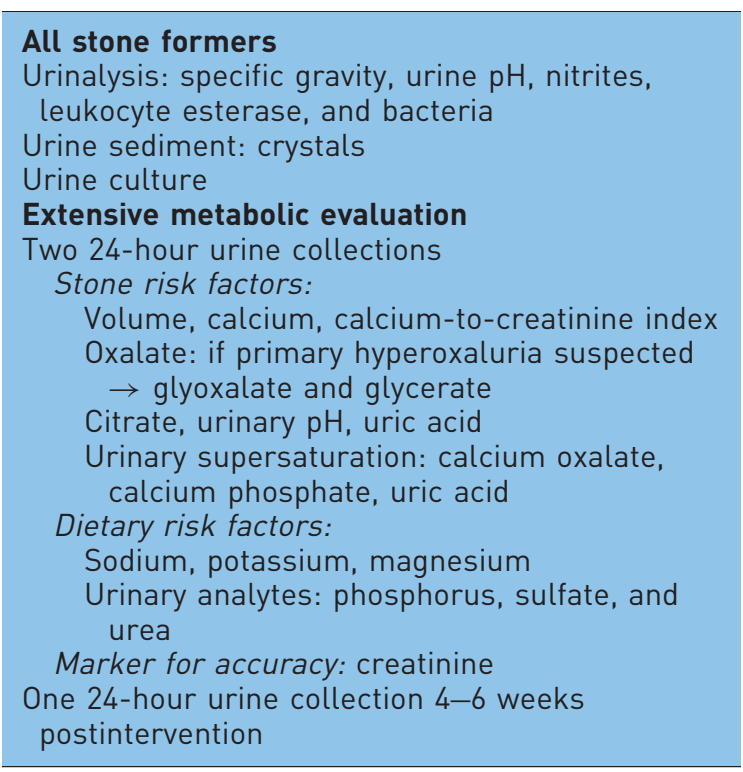

hypercalcemia and kidney stones, and an elevated serum angiotensin-converting enzyme (ACE) and calcitriol may provide supportive evidence of this disorder.

\section{Urine tests}

A simple clean catch urinalysis can be very informative and should be performed for all stone formers (Table 2). The specific gravity of urine reflects the general state of hydration of a patient; chronically volume depleted patients will demonstrate an elevated specific gravity, thereby implying an elevated stone risk. A simple urinalysis will also measure urinary $\mathrm{pH}$. Persistently low urinary $\mathrm{pH}(<5.5)$ increases the risk for uric acid calculi formation. A persistently elevated $\mathrm{pH}(>6.5)$ can be associated with distal RTA or the presence of urease-producing bacteria. Infection is supported with the concomitant presence of nitrites, leukocyte esterase, and bacteria.

If infection is suspected, a urine culture can be instructive. The results of the urine culture can demonstrate the presence of a urea-splitting organism, such as Proteus, Pseudomonas, or Klebsiella, all of which may be associated with the formation of struvite calculi.

Microscopic examination of the urine sediment can identify crystals that can predict the stone composition formed by the patient. Hexagonal crystals are pathognomonic for cystinuria [Prezioso et al. 2007]. Rectangular 'coffin-lid' 
Table 3. Indications for extensive metabolic evaluation.

Stone recurrence
Select first-time stone formers
Solitary kidney
Renal insufficiency
Residual stone burden
High risk for recurrence
Intestinal disease, chronic diarrhea
Pathologic skeletal fractures, osteoporosis
Gout, uric acid stones
Cystine stones, infection stones
All children

crystals indicate struvite calculi. In some patients, tetrahedral 'envelope' crystals can be detected which are present in the setting of calcium oxalate stones.

There are certain stone formers who should undergo a more extensive evaluation than that detailed above. Such patients include those who are recurrent stone formers, as they may have either persistent or severe metabolic abnormalities that can be treated medically. Other, first-time stone formers who should undergo a more extensive evaluation include those harboring large-volume residual stones, those at higher risk for recurrence, and those where further stone events could be detrimental (Table 3 ). In addition, pediatric stone formers may be more likely to have an underlying metabolic disturbance and therefore should also undergo a more complete investigation [DeFoor et al. 2006, 2008].

The 24-hour urine collection test is the mainstay of the comprehensive metabolic evaluation. A commonly encountered concern when performing a 24-hour urine study is whether one or two collections should be performed. Pak and associates have recommended a single 24 -hour collection for the diagnosis of metabolic conditions that increase risk of stone formation [Pak et al. 2001]. They found high reproducibility of stone risk factors in repeated samples. However, Parks and associates compared two separate 24-hour urine collections and found disparities great enough between the samples that clinical care may have been altered within nearly $70 \%$ of the comparisons [Parks et al. 2002]. We advise two 24-hour urine collections on initial evaluation to maximize the diagnostic yield. If metabolic therapy is initiated, a single 24-hour collection should be done 4-6 weeks after the initiation of treatment, to ensure that the desired effect has been achieved.

Any urinary tract infection should be treated prior to collection, as it may induce hypocitraturia and an elevated urinary $\mathrm{pH}$, potentially confounding the test results. In general, a 24-hour urine collection should not be performed in the course of an acute stone event, as the patient's routine lifestyle and dietary habits are altered [Begun et al. 1997]. Decisions regarding the management of medical therapy during the 24-hour urine collection should be patient and drug specific [Begun et al. 1997; Levy et al. 1995].

Stone risk factors. Low urine volume is one of the most common metabolic abnormalities encountered among stone formers. In general, a stone former should be encouraged to maintain a urine volume of 2 liters per day [Porena et al. 2007]. Low dietary fluid intake is the most common cause of this abnormality, although fluid losses by other mechanisms, such as chronic diarrheal states, can also result in low urine volume. This condition may coexist with hypocitraturia, another risk factor for stone formation.

Hypercalciuria in a patient with normal serum calcium may be considered idiopathic. Hypercalciuria may be defined as urine calcium excretion greater than $200 \mathrm{mg}$ per day in women and greater than $250 \mathrm{mg}$ per day in men; alternative methods of quantification include indexing calcium excretion to creatinine, in which case less than $140 \mathrm{mg} / \mathrm{g}$ creatinine is considered to be normal, or indexing to body weight, in which case less than $4 \mathrm{mg} / \mathrm{kg}$ for either sex would be considered to be normal. In some cases, idiopathic hypercalciuria may be due to an absorptive phenomenon: the patients absorb and excrete an elevated fraction of dietary calcium. In other cases, idiopathic hypercalciuria may be due to reduced renal tubular calcium reabsorption. Resorptive calciuria, a third type of hypercalciuria, is a manifestation of hyperparathyroidism, which occurs through a process of increased intestinal absorption of calcium, increased resorption of bone, and increased renal synthesis of $1,25(\mathrm{OH})_{2}$ vitamin $\mathrm{D}$. Other less common medical conditions can also cause hypercalciuria such as certain malignancies (multiple myeloma, lymphoma, leukemia, metastatic disease processes), sarcoidosis, Paget's disease, 
hyperthyroidism, vitamin $\mathrm{D}$ intoxication, and milk-alkali syndrome [Begun et al. 1997].

Hyperoxaluria ( $>40 \mathrm{mg}$ per day) is most commonly idiopathic. However, enteric hyperoxaluria should be suspected in patients with underlying small bowel pathology, such as surgical bowel resection or inflammatory bowel disease. Hyperoxaluria can often be found accompanying other stone risk factors such as low urine volume, low urine citrate, and low urine magnesium. A diagnosis of primary hyperoxaluria is suspected if urine oxalate is greater than $100 \mathrm{mg}$ per day. If suspected, the urine should also be tested for glyoxalate and glycerate [Leumann and Hoppe, 2001].

Hypocitraturia ( $<450 \mathrm{mg}$ in men, $<550 \mathrm{mg}$ in women per day) is present in over $30 \%$ of stone patients. In $50 \%$ of cases it coexists with other metabolic abnormalities and in up to $10 \%$ of cases it exists alone [Coe and Kavlach, 1974]. Citrate is protective against stone formation because it forms soluble complexes with calcium which inhibits crystal nucleation and growth [Heilberg and Schor, 2006; Seltzer et al. 1996]. Most commonly hypocitraturia is idiopathic, although distal RTA, chronic diarrhea, urinary tract infection, and thiazide medication can also induce hypocitraturia.

Urine $\mathrm{pH}$ should be between 5.8 and 6.2. Uric acid calculi can form when the urine $\mathrm{pH}$ is below the $\mathrm{pKa}$ of uric acid (5.75). The crystallization process of cystine is also $\mathrm{pH}$ dependent ( $\mathrm{pKa}$ of cystine is 8.3). There are some situations, though, where an increased urinary $\mathrm{pH}$ may promote, rather than inhibit, stone formation. Calcium phosphate stones form in a pH-dependent manner: distal RTA, which is associated with calcium phosphate stones, should be suspected when urinary $\mathrm{pH}$ is above 6.5. The presence of urea-splitting bacterial infection will raise urine $\mathrm{pH}$ above 8 , and should be suspected if such supraphysiologic $\mathrm{pH}$ measurements are present.

Hyperuricosuria ( $>800 \mathrm{mg}$ in men and $>750 \mathrm{mg}$ in women per day) is present in up to $35 \%$ of metabolic evaluations, although it is also coexistent with other abnormalities. Up to $20 \%$ of these patients will have calcium oxalate calculi [Begun et al. 1997]. The etiology of hyperuricosuric calcium oxalate nephrolithiasis may be due to either a heterogeneous nucleation process and/or a consumption of inhibitors. A gouty diathesis, Lesch-Nyhan syndrome, tumor lysis syndrome, myeloproliferative disorders, and an animal-protein-rich diet are all associated with hyperuricosuria and an increased risk of stone disease.

Urinary supersaturation is a measure of the likelihood of stone-forming salts to form a crystal complex, which can ultimately become a stone. Supersaturation calculations for salts, such as calcium oxalate, calcium phosphate, and uric acid, can reliably predict stone formation. The supersaturation value depends on the concentration of dissolved salt relative to its solubility. It is calculated using the total concentration of urinary sodium, potassium, calcium, magnesium, ammonia, phosphate, sulfate, oxalate, citrate, uric acid, pyrophosphate, carbon dioxide, chloride, and $\mathrm{pH}$.

Dietary risk factors. Increased levels of urinary sodium ( $>150 \mathrm{mg}$ per day) will increase urinary calcium excretion, and may also blunt the hypocalciuric effects of thiazide medications. Urinary calcium excretion increases by $25 \mathrm{mg}$ for every $100 \mathrm{mmol}$ increase in dietary sodium [Heilberg and Schor, 2006]. Sodium also reduces citrate excretion and increases cystine excretion [Porena et al. 2007].

Low potassium levels can promote hypocitraturia [Yachantha et al. 2009]. Urine potassium levels should be between 20 and $100 \mathrm{mmol}$ per day. Bowel diseases with chronic diarrhea, diuretic usage, or laxative abuse are all associated with low urinary potassium levels. Urine potassium levels may also be markers of medical therapy as the patient's urine potassium should increase following initiation of potassium citrate treatment and absence of an increase may be a marker of noncompliance.

Magnesium is an inhibitor of stone formation and urine excretion of this cation should be between 30 and $120 \mathrm{mg}$ per day. Magnesium can complex with renal oxalate, and supplementation with magnesium may increase urinary citrate excretion by decreasing its tubular reabsorption. Poor nutritional status, laxative abuse, and certain malabsorption syndromes are all associated with low magnesium levels. Low magnesium levels are found in up to $7 \%$ of stone formers is commonly associated with hypocitraturia and low urine volume [Preminger, 1994]. 
Urinary creatinine confirms the completeness of the 24-hour urine collection. In normal situations, creatinine excretion should be between 20 and $27 \mathrm{mg} / \mathrm{kg}$ in males and 14 and $21 \mathrm{mg} / \mathrm{kg}$ in females. Creatinine excretion should be consistent from day to day, and significant variations in this value should alert the physician that the days in question are not directly comparable due to either over or under collection of the specimen.

Increased protein consumption increases stone risk. Urinary analytes, such as phosphorus, sulfate, and urea, can be markers of the amount of animal protein consumption.

\section{Medical therapy of stone formers}

Medical therapy has been demonstrated to significantly decrease stone recurrence rates and may be cost effective as well [Lotan et al. 2005]. There are general recommendations that all stone formers should follow, regardless of diagnosis, to decrease stone recurrence rate. Stone formers should drink enough fluid to maintain a urine output of 2 liters per day. A low urine volume is among the most common metabolic abnormality found in stone formers and this is a modifiable risk factor [Porena et al. 2007]. A strict low-salt diet is also advised, as elevated sodium excretion in the urine can induce or exacerbate hypercalciuria. Increased sodium excretion can also blunt effectiveness of a thiazide-type medication used to treat hypercalciuria. Moderate animal protein intake is recommended, as purine gluttony is a known risk factor for uric acid stone formation. Lastly, intake of a normal recommended daily allowance of calcium ( $1200 \mathrm{mg}$ per day) is advised. A common misconception among stone formers is that a lowcalcium diet is a treatment for calcium stone disease. However, such a diet can actually increase stone risk, as demonstrated in a randomized, controlled trial performed by Borghi and colleagues [Borghi et al. 2002].

In addition to these generic recommendations for all stone formers, recommendations are based on the results of the workup discussed. Treatment of the diagnosed underlying etiology of the stone disease should be pursued.

Hyperparathyroidism causes resorptive hypercalciuria as elevated levels of parathyroid hormone stimulates uptake of calcium from the gut. Primary hyperparathyroidism is usually treated surgically. Idiopathic hypercalciuria can be treated with a thiazide-type medication [Lipkin and Shah, 2009]. Whether the etiology of idiopathic hypercalciuria is absorptive or resorptive, thiazide medications will increase tubular reabsorption of calcium in the kidney, thereby reducing the degree of calcium in the urine. Supplemental potassium may be necessary for patients receiving thiazide therapy, as these medications may promote hypokalemia, which can induce an intracellular acidosis and hypocitraturia.

Primary hyperoxaluria is an hereditary condition that is associated with stone disease. High-dose pyridoxine, magnesium, orthophosphate, glycosaminoglycans, and hydration are all used to manage this condition. Ultimately, however, liver and kidney transplantation is required for cure [Cochat and Basmaison, 2000]. Enteric hyperoxaluria can also occur for many different reasons but malabsorption or malnutrition are the most common causes. This can occur as a result of intestinal disease and/or chronic diarrhea. In addition, these patients often have great intestinal losses that result in low urine volume contributing an additional risk factor for kidney stone formation. The central treatment point to reduce urinary oxalate is dietary modification, hydration, calcium supplementation, magnesium, and reduction of other coexistent risk factors [Penniston and Nakada, 2009]. Cholestyramine and potassium citrate is also sometimes used. Hyperoxaluria can also be idiopathic.

Citrate therapy is used for many metabolic abnormalities that are associated with an increased risk of stone disease. It is used to raise the urine $\mathrm{pH}$ in uric acid stone formers. Citrate itself is an inhibitor of stone formation and hypocitraturia is among the most common metabolic abnormalities that are discovered in stone formers [Coe and Kavlach, 1974]. Hypocitraturia is often idiopathic, although other disease states, such as distal RTA, hypokalemia, chronic diarrhea, urinary tract infection, thiazide medication, and a low-alkali, high-protein diet can induce this disorder [Zuckerman and Assimos, 2009]. Again, hypocitraturia should be addressed by treating the underlying abnormality, but citrate replacement therapy is often the mainstay of therapy for this situation, such as those patients with distal RTA in which profound hypocitraturia is present. Dietary modifications with lemonade and other citrus-juice-based therapies can also help increase citrate in the urine [Zuckerman 
and Assimos, 2009]. One caveat to citrate therapy is to maintain vigilance for calcium phosphate stone formation, as the alkali nature of citrate medication will raise urinary $\mathrm{pH}$.

Finally, the presence of urease-producing bacteria, which are associated with struvite stone formation, should be addressed. Elimination of the infection is important, and it should be noted that eradication of the bacteria cannot be achieved if a stone burden is present. The central components of therapy for such patients are both surgical stone clearance as well as antimicrobial treatment of the offending bacteria.

\section{Conclusion}

Nephrolithiasis poses a significant social and financial burden. However, the impact of this disease can be mitigated by the appropriate metabolic evaluation of stone formers, in order to identify risk factors for recurrent stone events. Once such a workup is complete, attention may be focused on the medical treatment of these abnormalities. Ultimately, these efforts will reduce the deleterious effects that this prevalent disease has both on individual patient quality of life as well as the resource-constrained public health system.

\section{Conflict of interest statement}

The authors declare that there is no conflict of interest.

\section{References}

Begun, F.P., Foley, W.D., Peterson, A. and White, B. (1997) Patient evaluation laboratory and imaging studies, In: Resnick, M. (ed.). The Urologic Clinics of North America, Urolithiasis, Vol. 24, W.B. Saunders Company: Philadelphia, PA, pp. 97-116.

Borghi, L., Schianchi, T., Meschi, T., Guerra, A., Allegri, F., Maggiore, U. et al. (2002) Comparison of two diets for the prevention of recurrent stones in idiopathic hypercalciuria. $N$ Engl $\mathcal{F}$ Med 346: 77-84.

Cochat, P. and Basmaison, O. (2000) Current approaches to the management of primary hyperoxaluria. Arch Dis Child 82: 470-473.

Coe, F.L. and Kavlach, A.G. (1974) Hypercalciuria and hyperuricosuria in patients with calcium nephrolithiasis. N Engl f Med 291: 1344.

DeFoor, W., Asplin, J., Jackson, E., Jackson, C., Reddy, P., Sheldon, C. et al. (2006) Urinary metabolic evaluations in normal and stone forming children. f Urol 176: 1793-1796.

DeFoor, W., Minevich, E., Jackson, E., Reddy, P., Clark, C., Sheldon, C. et al. (2008) Urinary metabolic evaluations in solitary and recurrent stone forming children. F Urol 179: 2369-2372.

Eisner, B.H., Ahn, J. and Stoller, M.L. (2009) Differentiating primary from secondary hyperparathyroidism in stone patients: the "thiazide challenge". F Endourol 23: 191-192.

Evan, A.P., Lingeman, J., Coe, F., Shao, Y., Miller, N., Matlaga, B. et al. (2007) Renal histopathology of stone-forming patients with distal renal tubular acidosis. Kidney Int 71: 795-801.

Heilberg, I.P. and Schor, N. (2006) Renal stone disease: causes, evaluation, and medical treatment. Arq Bras Endocrinol Metab 50: 823-831.

Leumann, E. and Hoppe, B. (2001) The primary hyperoxalurias. F Am Soc Nephrol 12: 1986-1993.

Levy, F.L., Adams-Huet, B. and Pak, C.Y.C. (1995) Ambulatory evaluation of nephrolithiasis: an update of a 1980 protocol. Am f Med 98: 50-59.

Lipkin, M. and Shah, O. (2009) Medical therapy of stone disease: from prevention to promotion of passage options. Curr Urol Rep 10: 29-34.

Lotan, Y., Cadeddu, J.A. and Pearle, M.S. (2005) International comparison of cost effectiveness of medical management strategies for nephrolithiasis. Urol Res 33: 223-230.

Odvina, C.V. and Pak, C.Y.C. (2007) Medical evaluation of stone disease, In: Stoller, M.L. and Men, M.V. (eds). Urinary Stone Disease: A Practical Guide to Medical and Surgical Management (Current Clinical Urology, Vol. 1), Humana Press Inc: Totowa, NJ.

Pak, C.Y.C., Britton, F., Peterson, R., Ward, D., Northcutt, C., Breslau, N.A. et al. (1980) Ambulatory evaluation of nephrolithiasis. Classification, clinical presentation and diagnostic criteria. $\mathrm{Am} \mathcal{F} \mathrm{Med}$ 69: 19-30.

Pak, C.Y.C., Peterson, R. and Poindexter, J.R. (2001) Adequacy of a single stone risk analysis in the medical evaluation of urolithiasis. F Urol 165: 378-381.

Parks, J.H., Goldfisher, E., Asplin, J.R. and Coe, F.L. (2002) A single 24-hour urine collection is inadequate for the medical evaluation of nephrolithiasis. 7 Urol 167: 1607-1612.

Pearle, M.S., Calhoun, E.A. and Curhan, G.C., for the Urologic Disease of America Project (2005) Urologic Diseasesin America Project: urolithiasis. $\mathcal{F}$ Urol 173: 848-857.

Penniston, K.L. and Nakada, S.Y. (2009) Effect of dietary changes on urinary oxalate excretion and calcium oxalate supersaturation in patients with hyperoxaluric stone formation. Urology 73: 484-489.

Porena, M., Guiggi, P. and Micheli, C. (2007) Prevention of stone disease. Urol Int 79(Suppl 1): 37-46.

Preminger, G.M. (1994) Medical evaluation and treatment of nephrolithiasis. Semin Urol 12: 51. 
Prezioso, D., Di Martino, M., Galasso, R. and Iapicca, G. (2007) Laboratory assessment. Urol Int 79(Suppl 1): 20-25.

Seltzer, M.A., Low, R.K., McDonald, M., Shami, G.S. and Stoller, M.L. (1996) Dietary manipulation with lemonade to treat hypocitraturic calcium nephrolithiasis. F Urol 156: 907-909.

Stamatelou, K.K., Francis, M.E., Jones, C.A., Nyberg, L.M. and Curhan, G.C. (2003) Time trends in reported prevalence of kidney stones in the United States: 1976-1994. Kidney Int 63: 1817-1823.

Yachantha, C., Hossain, R.Z., Yamakawa, K., Sugaya, K., Tosukhowong, P., Ogawa, Y. et al. (2009) Effect of potassium depletion on urinary stone risk factors in Wistar rats. Urol Res 37: 311-316.

Zuckerman, J.M. and Assimos, D.G. (2009)

Hypocitraturia: pathophysiology and medical management. Rev Urol 11: 134-144.
Visit SAGE journals online http://tau.sagepub.com

QSAGEJOURNALS 